\title{
Predicting malignancy in thyroid nodules: feasibility of a predictive model integrating clinical, biochemical, and ultrasound characteristics
}

Justyna Witczak ${ }^{1,4^{*}+}$, Peter Taylor ${ }^{1,4 \dagger}$, Jason Chai ${ }^{4}$, Bethan Amphlett ${ }^{4}$, Jean-Marc Soukias ${ }^{2}$, Gautam Das ${ }^{4}$, Brian P. Tennant ${ }^{3}$, John Geen ${ }^{3,5}$ and Onyebuchi E. Okosieme $e^{1,4}$

\begin{abstract}
Background: Although the majority of thyroid nodules are benign the process of excluding malignancy is challenging and sometimes involves unnecessary surgical procedures. We explored the development of a predictive model for malignancy in thyroid nodules by integrating a combination of simple demographic, biochemical, and ultrasound characteristics.

Methods: Retrospective case-record review. We reviewed records of patients with thyroid nodules referred to our institution from 2004 to 2011 ( $n=536$; female $84 \%$, mean age 51 years). All malignancy was proven histologically while benign disease was either confirmed histologically, or on cytology with minimum 36-month observation period. We focused on the following predictors: age, sex, smoking status, thyroid hormones (FT4 and TSH) and nodule characteristics on ultrasound. Variables were included in a multivariate logistic regression and bootstrap analyses were used to confirm results.

Results: Independent predictors of malignancy in the fully adjusted model were TSH (OR 1.53, $95 \% \mathrm{Cl} 1.10,2.12, p=0.01$ ), male gender (OR 3.45, $95 \% \mathrm{Cl} 1.33,8.92, p=0.01$ ), microcalcifications (OR 6.32, $95 \% \mathrm{Cl} 2.82,14.1, p<0.001$ ), and irregular nodule margins (OR 5.45, $95 \% \mathrm{Cl} 1.61,18.6, p=0.006)$ Bootstrap analyses strengthened these associations and a parsimonious analysis consisting of these variables and age-group demonstrated an area under the curve of 0.77 . A predictive score was sensitive (86.9\%) at low scores and highly specific (94.87\%) at higher scores for distinguishing benign from malignant disease.
\end{abstract}

Conclusions: A predictive model for malignancy using a combination of clinical, biochemical, and radiological characteristics may support clinicians in reducing unnecessary invasive procedures in patients with thyroid nodules.

Keywords: Thyroid cancer, Thyroid nodule, Diagnosis, Predictive model, TSH, Ultrasound

\footnotetext{
*Correspondence: WitczakJK@cardiff.ac.uk

${ }^{\dagger}$ Equal contributors

${ }^{1}$ Centre for Endocrine and Diabetes Science, Institute of Molecular and

Experimental Medicine, Cardiff University School of Medicine, University Hospital Wales, Cardiff CF14 4XN, UK

${ }^{4}$ Endocrinology and Diabetes Department, Prince Charles Hospital, Cwm Taf

Health Board, Gurnos Estate Merthyr Tydfil CF47 9DT, UK

Full list of author information is available at the end of the article
} 


\section{Background}

Thyroid cancer is the most common endocrine malignancy accounting for more deaths than all other endocrine cancers combined [1]. The incidence of thyroid cancer has continued to increase worldwide, partly due to greater diagnostic activity, as well as a true increase in disease occurrence $[2,3]$. The differentiated thyroid cancers, papillary and follicular, are the most prevalent histological types with papillary cancer accounting for $40-70 \%$ of all cases of thyroid cancer [1]. In recent years small papillary microcarcinomas, less than one $\mathrm{cm}$ in size, are now increasingly detected due to the growing use of modern imaging techniques such as ultrasound, computerised tomography or positron emission tomography (PET) scans [4]. Although differentiated thyroid cancers generally carry a favourable prognosis affected individuals suffer considerable morbidity, both from the disease as well as from side effects of therapy. In most instances treatment with thyroidectomy and radioactive iodine ablation will incur the need for lifelong thyroid hormone therapy [5].

Patients with thyroid cancer commonly present with clinically or radiologically detectable thyroid nodules $[6,7]$. The prevalence of palpable thyroid nodules is estimated at about 4-7 \% of the adult population in iodine-sufficient areas [6] with higher figures reported in women, older patients, and in studies based on ultrasound scan examination [6-8]. Yet despite the high frequency of thyroid nodules only about $5-10 \%$ of such nodules are malignant $[6,7]$. Fine needle aspiration biopsy (FNAB) and histology is the gold standard for diagnosing thyroid cancer but up to $25 \%$ of biopsies performed in practice are non-diagnostic [9]. Patients with non-diagnostic biopsies often undergo repeat biopsies and in some cases proceed to a diagnostic thyroidectomy with its accompanying risks of permanent hypoparathyroidism and recurrent laryngeal nerve damage [10].

The challenge for the clinician is thus to predict which thyroid nodules are malignant as patients with benign nodules can be spared unnecessary intervention. A number of simple clinical characteristics have traditionally been associated with an increased malignancy risk including male gender, age $<30$ years or $>60$ years, a family history of thyroid cancer, previous exposure to head and neck irradiation, and a firm, hard, rapidly enlarging nodule with compressive symptoms such as dysphagia, dysphonia and breathlessness [6]. Ultrasound examination of the thyroid is also a useful adjunct to clinical assessment, and nodule characteristics that carry a high risk of malignancy include hypoechogenicity, microcalcifications, irregular margins, solid composition, and single nodularity [11]. However when considered individually none of these features are sufficiently sensitive to guide clinical decisions [12].
In recent years serum thyrotropin (TSH) has emerged as an independent predictor of malignancy in patients with thyroid nodules [13, 14]. TSH is a thyroid growth factor and in animal experiments stimulates thyroid cell proliferation through cyclic AMP mediated pathways, thereby promoting thyroid malignancy [15]. Furthermore, levothyroxine-induced TSH suppression remains a therapeutic cornerstone for preventing cancer recurrence following thyroidectomy and radioactive iodine treatment [16]. In observational studies a direct relationship has been shown between TSH concentrations and thyroid cancer risk and other adverse outcomes even at TSH levels within the euthyroid range [17, 18]. In addition increased TSH concentration is associated with a more advanced stage of thyroid cancer at presentation [19] adding credence to its pivotal role in the development and progression of thyroid cancer.

Thus, the prediction of thyroid cancer in patients with thyroid nodules involves multiple clinical, biochemical, and radiological risk factors and a predictive model is unlikely to be satisfactorily achieved by a single set of predictors. While most studies to date have focused on single risk factors, whether clinical, radiological, or biochemical, only a few studies have evaluated these factors in combination. A robust predictive model incorporating a combination of simple easily obtainable clinical, laboratory and radiological risk factors may offer adequate sensitivity to serve as a pragmatic decision aid to support clinicians in practice. The aim of this study therefore was to explore the potential of developing a predictive model for the risk of thyroid cancer in patients with thyroid nodules using demographic, clinical, laboratory and ultrasound predictors.

\section{Methods \\ Patients}

We retrospectively reviewed the records of patients referred for evaluation of thyroid nodules at our institution between January 2004 and December 2011. Patients with thyroid nodules were identified from histopathology records $(n=602)$. We excluded patients with inadequate clinical or laboratory information and patients in whom a final diagnosis was not reached $(n=66)$. All diagnoses of malignancy were proven histologically after thyroidectomy or surgical biopsy $(n=50)$. Benign disease was either confirmed histologically in patients following thyroidectomy or surgical biopsy $(n=362)$ or was based on a negative fine needle aspiration cytology and a minimum clinical observation period of 36 months in those who did not undergo surgery $(n=124)$. We evaluated the following candidate predictors: age, gender, smoking status, thyroid hormone status i.e. serum free thyroxine (FT4) and serum TSH, and thyroid ultrasound characteristics (hypoechogenicity, microcalcifications, irregular 
margins, spherical shape, and single nodularity and solid composition). Approval to undertake the study was received from the NHS Research and Ethics Committee for Wales (reference 12/WA/0334) and the Cwm Taf University Health Board Research and Development Office (reference CT/278/101463/12).

\section{Statistical analysis}

To select suitable predictors in the model all apriori candidate predictors were entered initially into a univariate analysis and then all variables were included in a multivariate logistic regression to analyse the odds of developing malignancy. The relevant factors and their weighting were then built into a final model. TSH and FT4 levels were categorized into tertiles. As the risk of malignancy is increased in young adults as well as the elderly we coded age as 1 if the age was less than 30 years or more than 60 years while patients aged 3060 years were coded as 0 . Smokers were defined as never smoked, previous smokers, or current smokers. Ultrasound characteristics such as size, echogenicity and nodule composition were utilised as ordered categorical variables. Logistic regression was undertaken to assess the odds of malignancy for each of these variables. In addition we explored for interaction between TSH level and age-group and TSH and gender using likelihood ratio tests. We then modelled in a parsimonious approach all variables associated with malignancy in univariate or multivariate analysis maintaining age-group and gender as forced variables. Confirmatory bootstrap analysis was undertaken with 1000 iterations using age-group and gender as strata. These variables were taken forward for area under the receiver operating characteristic (ROC) using the "lroc" command in STATA. A risk score was then generated based on identified associations and the magnitude of their effect. All statistical analysis was undertaken using STATA version 12 (STATACORP, College Station, TX, USA).

\section{Results}

\section{Baseline characteristics}

We identified 602 patients from the thyroid histopathological records. Of these, 48 (8\%) did not have a final cytological or histological diagnosis and clinical records were irretrievable in 18 (3\%) patients leaving a final sample size of 536 for analysis. Data on smoking was missing for 81 individuals while 22 individuals had missing data for TSH. The baseline characteristics of these patients is summarised in Table 1. As expected the majority of our cohort was female, young to middle age, with a $9 \%$ malignancy detection rate. The histological subtypes comprised papillary (69\%), follicular (8\%), hurtle cell (4\%), anaplastic (6\%), lymphoma (6\%), and medullary cancer $(6 \%)$. Of the 536 patients $70(13 \%)$ had indeterminate cytology including atypical cytology (Thy3a) and suspected follicular neoplasm (Thy3f) [5]; of these 5 patients $(7 \%)$ had a final diagnosis of malignancy.

\section{Predictors of malignancy}

Independent predictors of malignancy in the fully adjusted model were TSH group (OR 1.53, $95 \%$ CI 1.10, 2.12, $p=0.01$ ), male gender (OR 3.45, $95 \%$ CI 1.33, $8.92, p=0.01$ ), the presence of microcalcifications (OR 6.32, $95 \%$ CI 2.82, 14.1, $p<0.001)$, and irregular margins (OR 5.45, 95 \% CI 1.61, 18.6, $p=0.006$ ) (Table 2). The association between TSH levels and malignancy was only marginally attenuated when adjusting for FT4. In this relationship, we found no evidence of interaction between TSH levels and age-group $(p=0.91)$ or gender $(p=0.64)$. Bootstrap analysis confirmed the key associations with all associations remaining statistically significant (Table 3). A parsimonious analysis consisting variables associated with malignancy and forced variables demonstrated an area under the curve of 0.77 (Additional file 1: Figure S1).

\section{Score generation}

Based on our above analysis we generated a score to predict malignancy. We used TSH $>2.5=1$, Age-group $<30$ or $>60=1$, presence of microcalcification $=2$, male gen$\operatorname{der}=2$, irregular nodule margins $=2$, mixed echogenicity $=1$, and hypoechogenicity $=2$. Overall, scores $>4$ were highly sensitive $(86.9 \%)$ whereas $>7$ were highly specific $(94.87 \%)$ for malignancy (Table 4$)$. Out of 70 patients with indeterminate cytology, 40 patients (57\%) had a score of 2 or less of which 2 patients $(5 \%)$ had a final diagnosis of malignancy while 21 patients (30\%) with a score of 1 or less all had a final diagnosis of benign nodules. Thus in patients with indeterminate cytology, the negative predictive value of a score of 2 or less was $95-100 \%$.

\section{Discussion}

As in other countries we have previously reported an increase in the incidence of thyroid cancer in Wales, a trend which appears predominantly driven by the growing detection of papillary thyroid cancers [20]. In parallel we have also seen a steady increase in the number of thyroid surgical procedures performed for malignant thyroid disease in the same population [21]. The patient with a thyroid nodule thus presents a challenge as it is crucial to accurately identify the small proportion of malignant cases and at the same time avoid subjecting individuals with benign nodules to unwarranted surgical interventions with lifelong repercussions. A robust predictive model for malignancy could therefore support clinicians and patients in arriving at an informed decision regarding the urgency and scope of intervention 
Table 1 Baseline characteristics of patients

\begin{tabular}{|c|c|c|c|}
\hline Characteristics & Benign & Malignant & Total \\
\hline Number & 486 & 50 & $536(100 \%)$ \\
\hline Male sex (\%) & $67(13.8 \%)$ & $19(38.0 \%)$ & $86(16.0 \%)$ \\
\hline Age (years) & $51.2(15.9)$ & $50.9(15.9)$ & $51.2(15.9)$ \\
\hline \multicolumn{4}{|l|}{ Smoking status } \\
\hline Never & $240(57.4 \%)$ & $23(62.1 \%)$ & $263(57.8 \%)$ \\
\hline Previous & $53(12.7 \%)$ & 3 (8.11 \%) & $56(12.3 \%)$ \\
\hline Current & $125(29.9 \%)$ & $11(29.7 \%)$ & $136(29.9 \%)$ \\
\hline \multicolumn{4}{|l|}{ Thyroid status } \\
\hline TSH (mU/l) & $1.20(0.50-2.00)$ & $1.95(1.13-2.90)$ & $1.40(0.70-2.40)$ \\
\hline FT4 (pmol/l) & $15.8(7.52)$ & $13.8(5.38)$ & $16.9(7.56)$ \\
\hline Nodule size (cm) & $2.91(1.63)$ & $2.99(1.44)$ & $2.91(1.61)$ \\
\hline Microcalcification & $61(12.5 \%)$ & $26(52.0 \%)$ & $87(16.2 \%)$ \\
\hline \multicolumn{4}{|l|}{ Nodule Composition } \\
\hline Cystic & $90(18.5 \%)$ & $6(12.0 \%)$ & $96(17.9 \%)$ \\
\hline Mixed & $53(10.9 \%)$ & $6(12.0 \%)$ & $59(11.0 \%)$ \\
\hline Solid & $343(70.6 \%)$ & 38 (76.0 \%) & 381 (71.1%) \\
\hline \multicolumn{4}{|l|}{ Echogenicity } \\
\hline Iso or hyper-echoic & $332(68.3 \%)$ & $25(50.0 \%)$ & $357(66.6 \%)$ \\
\hline Mixed & $59(12.1 \%)$ & $9(18.0 \%)$ & $68(12.7 \%)$ \\
\hline Hypo-echoic & 95 (19.6\%) & $16(32.0 \%)$ & $111(20.7 \%)$ \\
\hline \multicolumn{4}{|l|}{ Margins } \\
\hline Regular & $469(96.5 \%)$ & $43(86.0 \%)$ & $512(95.5 \%)$ \\
\hline Irregular & 17 (3.50 \%) & 7 (14.0\%) & 24 (4.5 \%) \\
\hline
\end{tabular}

Figures are mean (SD), number (\%) or median (IQR)

especially where FNAB is considered inconclusive or inadequate for analysis.

Our multivariate analysis confirms some well-established risk factors for thyroid malignancy including male gender, $\mathrm{TSH}>2.5 \mathrm{mU} / \mathrm{L}$, and typical ultrasound characteristics of microcalcification, irregular nodule margin, and solid nodule composition [22]. However, we found no association between age $(<30$ or $>60$ years) and risk of malignancy. The reason for this is unclear but may indicate a changing epidemiology with increasing detection of thyroid cancer

Table 2 Odds Of Malignancy In Univariate And Multivariate Analyses

\begin{tabular}{|c|c|c|c|c|}
\hline \multirow[b]{2}{*}{ Variable } & \multicolumn{2}{|c|}{ Univariate analysis $(n=536)$} & \multicolumn{2}{|c|}{ Multivariate analysis $(n=422)$} \\
\hline & OR $(95 \% \mathrm{Cl})$ & $p^{a}$ & OR $(95 \% \mathrm{Cl})$ & $p^{a}$ \\
\hline TSH - upper tertile & $1.52(1.19,1.92)$ & 0.001 & $1.53(1.10,2.12)$ & 0.01 \\
\hline FT4 - upper tertile & $0.82(0.66,1.02)$ & 0.08 & $0.84(0.61,1.15)$ & 0.28 \\
\hline Age-group ( $<30$ or $>60$ yrs) & $1.16(0.64,2.09)$ & 0.62 & $1.17(0.51,2.71)$ & 0.71 \\
\hline Male gender & $3.83(2.04,7.17)$ & $<0.001$ & $3.45(1.33,8.92)$ & 0.01 \\
\hline Smoker & $0.93(0.64,1.38)$ & 0.75 & $1.02(0.65,1.60)$ & 0.93 \\
\hline Size group & $0.79(0.55,1.15)$ & 0.23 & $0.78(0.45,1.37)$ & 0.40 \\
\hline Microcalcification & $7.55(4.07,14.0)$ & $<0.001$ & $6.32(2.82,14.1)$ & $<0.001$ \\
\hline Nodularity & $1.09(0.59,2.01)$ & 0.78 & $0.79(0.36,1.76)$ & 0.57 \\
\hline Hypo-echogenicity & $1.52(1.09,2.10)$ & 0.01 & $1.17(0.73,1.89)$ & 0.51 \\
\hline Irregular margins & $4.49(1.76,11.4)$ & 0.002 & $5.45(1.61,18.6)$ & 0.006 \\
\hline Solid composition & $1.24(0.82,1.87)$ & 0.31 & $1.05(0.62,1.77)$ & 0.84 \\
\hline
\end{tabular}

${ }^{a}$ Calculated using the Wald Test; Multivariate analysis is analysis adjusted for all variables shown in this table 
Table 3 Odds of malignancy using a parsimonius approach with confirmatory bootstrap analysis

\begin{tabular}{|c|c|c|c|c|}
\hline \multirow[b]{2}{*}{ Variable } & \multicolumn{2}{|c|}{ Multivariate Analysis $(N=514)$} & \multicolumn{2}{|c|}{ Bootstrap analysis $(N=514)$} \\
\hline & Odds Ratio $(95 \% \mathrm{Cl})$ & $\mathrm{p}^{\mathrm{a}}$ & Odds Ratio $(95 \% \mathrm{Cl})$ & $p^{a}$ \\
\hline TSH - upper tertile & $1.65(1.28,2.14)$ & $<0.001$ & $1.65(1.28,2.13)$ & $<0.001$ \\
\hline Age-group $<30$ or $>60$ years & $1.20(0.60,2.37)$ & 0.61 & $1.20(0.57,2.47)$ & 0.63 \\
\hline Male gender & $4.41(2.06,9.46)$ & $<0.001$ & 4.41 (1.97. 9.89) & $<0.001$ \\
\hline Microcalcification & $7.33(3.62,14.8)$ & $<0.001$ & $7.33(3.35,16.1)$ & $<0.001$ \\
\hline Echogenicity & $1.24(0.82,1.86)$ & 0.30 & $1.24(0.80,1.91)$ & 0.34 \\
\hline Irregular margins & $3.18(1.05,9.63)$ & 0.04 & $3.18(0.95,10.6)$ & 0.06 \\
\hline
\end{tabular}

${ }^{a}$ Calculated using the Wald Test, Multivariate analysis is analysis adjusted for all variables shown in this table

across all ages as shown in recent surveys from our population and others $[20,23]$. Thus the majority of the power for prediction was derived from the thyroid ultrasound findings although the inclusion of TSH and gender further improved the model.

Using a scoring system of 2-10 we were able to stratify the risk of malignancy in patients presenting with thyroid nodules. Our score had high specificity $(95 \%)$ at high levels $(>7)$ but only a small proportion $(4 \%)$ of our cohort fell into this category. Nonetheless our score would be particularly useful for risk stratification in individuals with inconclusive FNAB or where invasive investigations are considered unduly intrusive such as in the frail, elderly, or in pregnancy. In such scenarios higher scores would dictate an aggressive approach while lower scores would support clinicians in adopting a more conservative or "wait and watch" policy. In our sample a score of 2 or less was associated with a low probability of disease in patients with indeterminate cytology (negative predictive value 95-100 \%) and thus low scoring individuals with indeterminate cytology could have been safely managed conservatively.

A number of studies have developed predictive models for thyroid cancer in patients with thyroid nodules using

Table 4 Sensitivity, specificity and correct classifaction of malignancy based on predictive score

\begin{tabular}{llllrl}
\hline Cut-point & Sensitivity & Specificity & Correctly Classified & LR+ & LR- \\
\hline$(>=2)$ & $100 \%$ & $0.00 \%$ & $8.95 \%$ & 1.00 & \\
$(>=3)$ & $93.48 \%$ & $28.21 \%$ & $34.05 \%$ & 1.30 & 0.23 \\
$(>=4)$ & $86.96 \%$ & $52.78 \%$ & $55.84 \%$ & 1.84 & 0.25 \\
$(>=5)$ & $65.22 \%$ & $72.65 \%$ & $71.98 \%$ & 2.38 & 0.48 \\
$(>=6)$ & $45.65 \%$ & $87.82 \%$ & $84.05 \%$ & 3.75 & 0.62 \\
$(>=7)$ & $28.26 \%$ & $94.87 \%$ & $88.91 \%$ & 5.51 & 0.76 \\
$(>=8)$ & $23.91 \%$ & $97.86 \%$ & $91.25 \%$ & 11.19 & 0.78 \\
$(>=9)$ & $13.04 \%$ & $99.15 \%$ & $91.44 \%$ & 15.26 & 0.88 \\
$(>=10)$ & $4.35 \%$ & $99.79 \%$ & $91.25 \%$ & 20.35 & 0.96 \\
\hline
\end{tabular}

The positive likelihood ratio $(\mathrm{LR}+)$ is the ratio of the probability of a positive test among the truly positive subjects to the probability of a positive test among the truly negative subjects. The negative likelihood ratio (LR-) is the ratio of the probability of a negative test among the truly positive subjects to the probability of a negative test among the truly negative subjects a variety of approaches. Stojadinovic et al used Bayesian analysis to build a clinical decision aid based on a prospective analysis of 216 patients with thyroid nodules who underwent thyroidectomy [24]. Their model incorporated nodule size, FNAB cytology, and ultrasound characteristics, and was highly predictive of malignancy (AUC 0.88, 95 \% CI; 0.82-0.94). However this model was also based on the technique of electrical impedance scanning which is not routinely available in practice [24]. Other studies utilising similar Bayesian principles have reported various combinations of clinical and sonographic predictors such as age, gender, and ultrasound characteristics $[25,26]$. One study utilised a classification and regression tree (CART) classifier in a two-stage model (sensitivity $80 \%$, specificity $94.1 \%$ ) that included age, sex, and nodule size in the first stage and echogenicity and microcalcifications in the second stage [27].

Another approach used by others has been to develop risk stratification algorithms derived from multivariate analysis. Boelaert and co-workers calculated a predictive formula for malignancy in patients with nodules, using age, gender, goitre type, and serum TSH [14]. In their study a TSH in the upper third of the normal range was significantly associated with malignancy (OR $2.9195 \%$ CI $1.49,5.71)$ [14]. By means of a regression model Maia et al showed a strong risk of malignancy in patients aged $>39$ years and in those with suspicious ultrasound features (predictive accuracy, $81.7 \%$ ) [28]. Similar findings were reported in another study restricted to individuals with indeterminate or suspicious FNABs [29]. Elsewhere, Nixon et al applied 8 predictor variables including TSH, ultrasound characteristics, and FNA cytology to create a nomogram with a high discriminatory ability (concordance index, $91 \%$ ) [30]. One mathematical formula derived from ultrasound characteristics alone proved reliable for detecting non-follicular neoplasms (sensitivity, $86.5 \%$, specificity $92.3 \%$ ) [31]. However the utility of complex calculations and algorithms in busy clinical settings is still unclear.

The strength of our study lies in the simplicity and practical appeal of our predictive score as an office based tool derived from a combination of easily obtainable 
predictors. As a decision aid our score could inform clinicians and potentially limit the number of unnecessary invasive procedures undertaken in low-risk individuals with inconclusive or inadequate FNABs. Furthermore our data, sourced from a relatively large clinic population, is representative of patients with thyroid nodules encountered in everyday practice. The malignancy rate of $9 \%$ in our sample is consistent with the prevalence of malignancy in secondary care referral settings. However our study has some limitations. Although all cases of malignancy were diagnosed by surgical specimen histology some individuals with benign disease did not undergo surgery but relied on the FNAB and a three year clinical observation period for the final diagnosis. Nonetheless this approach reduced surgical selection bias allowing us to analyse a broader spectrum of patients with a greater percentage of low risk individuals. Data were not available on anti-TPO antibody status for the majority in patients therefore this was not included in models. Lastly our data was retrospective and will have to be considered preliminary pending confirmation in an externally validated prospective cohort.

\section{Conclusion}

In conclusion a predictive model for malignancy using a combination of simple accessible clinical, biochemical, and sonographic predictors could improve the management of thyroid nodules by supporting clinicians in reducing invasive surgical procedures in low risk patients with thyroid nodules.

\section{Additional file}

Additional file 1: Figure S1. Area under the curve for model predicting malignancy. Area under ROC curve $=0.7667$ (PDF $14 \mathrm{~kb}$ )

\section{Abbreviations}

AMP, Adenosine monophosphate; AUC, area under the curve; $\mathrm{Cl}$, Confidence interval; FNAB, Fine needle aspiration biopsy; FT4, Free thyroxinel; OR, Odds ratio; PET, Positron emission tomography; ROC, receiver operating characteristics; TSH, Thyroid stimulating hormone

\section{Acknowledgements}

None. No funding to declare.

\section{Availability of data and materials}

We are reluctant to share this data in a publicly accessible repository as this would breech patient confidentiality according to the terms of governance approval for the study: NHS Research and Ethics Committee for Wales (ref, 12NA/0334) and the Cwm Taf University Health Board Research and Development Office (ref, CT/278/101463/12).

\section{Authors' contributions}

WJ, TP and OOE were involved in study design, acquisition of data, analysis and interpretation of data and drafting and revising the manuscript. CJ and $A B$ were involved in data collection and analysis and manuscript drafting. SJ, $\mathrm{DG}, \mathrm{TB}, \mathrm{GJ}$ made contributions to study conceptions and design and manuscript revision. All authors gave their approval for the final version to be published and agree to be accountable for this work.

\section{Authors' information}

Dr Justyna Witczak is a Specialist Registrar in Diabetes and Endocrinology in Wales currently working as a Research Fellow at Cardiff University. Her research is supported by Lewis Thomas Gibbon Jenkins Fellowship from the Royal College of Physicians. Her main areas of interest are extracellular vesicles and their role in obesity driven inflammation as well as thyroid disorders.

Dr Peter Taylor is a Specialist Registrar in Diabetes and Endocrinology awarded the Welsh Clinical Academic Track Fellowship and currently completing his PhD at Cardiff University. His main areas of interest are thyroid disorders, epidemiology and medical statistics and he has extensive experience in working with large datasets.

\section{Competing interests}

The authors declare that they have no competing interests.

\section{Consent to participate}

Not required.

\section{Consent for publication}

All authors approved this manuscript for publication in the current form. The data from which our model was developed contains clinical details of individuals treated for thyroid cancer from a single health board. As our study was retrospective it was not feasible to seek individual participant consent and ethical approval was granted to proceed without consent on the agreement that patient identifiable information would remain confidential and would not be accessible to any individuals outside of the direct clinical care team of the participants.

As ours is a relatively small health board and thyroid cancer is not that common it is not inconceivable that individual participants could be identified from a spread sheet of clinical details based on basic clinical and demographic information such as age, gender, smoking history etc. Even if such individuals were not identifiable the perception of identification whether true or imagined may cause distress to affected individuals. Therefore the data is not going to be shared in a publicly available repository.

\section{Ethics approval}

Approval to undertake this study was received from the NHS Research and Ethics Committee for Wales (reference 12/WA/0334) and the Cwm Taf University Health Board Research and Development Office (reference CT/ 278/101463/12)

\section{Author details}

${ }^{1}$ Centre for Endocrine and Diabetes Science, Institute of Molecular and Experimental Medicine, Cardiff University School of Medicine, University Hospital Wales, Cardiff CF14 4XN, UK. ${ }^{2}$ Radiology Department, Prince Charles Hospital, Cwm Taf Health Board, Gurnos Estate Merthyr Tydfil CF47 9DT, UK. ${ }^{3}$ Clinical Biochemistry Department, Prince Charles Hospital, Cwm Taf Health Board, Gurnos Estate Merthyr Tydfil CF47 9DT, UK. ${ }^{4}$ Endocrinology and Diabetes Department, Prince Charles Hospital, Cwm Taf Health Board, Gurnos Estate Merthyr Tydfil CF47 9DT, UK. ${ }^{5}$ Faculty of Life Sciences and Education, University of South Wales, Glyntaff Campus, Pontypridd CF37 1DL, UK.

Received: 8 March 2016 Accepted: 19 May 2016

Published online: 25 May 2016

References

1. Monson JP. The epidemiology of endocrine tumours. Endocr Relat Cancer. 2000:7:29-36.

2. Vigneri R, Maladrino P, Vigneri P. The changing epidemiology of thyroid cancer: why is incidence increasing? Curr Opin Oncol. 2015;27:1-7.

3. Wartofsky L. Increasing world incidence of thyroid cancer: increased detection or higher radiation exposure? Hormones (Athens). 2010;9:103-8.

4. Pearce EN, Braverman LE. Papillary thyroid microcarcinoma outcomes and implications for treatment. J Clin Endocrinol Metab. 2004;89:3710-2.

5. Perros P, Boelaert K, Colley S, Evans C, Evans RM, Gerrard Ba G, et al. British Thyroid Association. Guidelines for the management of thyroid cancer. Clin Endocrinol (Oxf). 2014:81 Suppl1:1-122.

6. Hegedüs L. The thyroid nodule. N Engl J Med. 2004;351:1764-71, 
7. De Martino E, Pirola I, Gandossi E, Delbarba A, Cappelli C. Thyroid nodular disease: an emerging problem. Minerva Endocrinol. 2008;33:15-25.

8. Ezzat S, Sarti DA, Cain DR, Braunstein GD. Thyroid incidentalomas. Prevalence by palpation and ultrasonography. Arch Intern Med. 1994;154: 1838-40.

9. Chow LS GH, Goellner JR, van Heerden JA. Nondiagnostic thyroid fine-needle aspiration cytology: management dilemmas. Thyroid. 2001; 11:1147-51.

10. Burch HB. Evaluation and management of the solid thyroid nodule. Endocrinol Metab Clin North Am. 1995;24:663-710.

11. Frates MC, Benson CB, Doubilet PM, Kunreuther E, Contreras M, Cibas ES, et al. Prevalence and distribution of carcinoma in patients with solitary and multiple thyroid nodules on sonography. J Clin Endocrinol Metab. 2006;91:3411-7.

12. Frates MC, Benson CB, Charboneau JW, Cibas ES, Clark OH, Coleman BG, et al. Management of thyroid nodules detected at US: Society of Radiologists in Ultrasound consensus conference statement. Radiology. 2005;237:794-800

13. Fiore $E$, Vitti P. Serum TSH and risk of papillary thyroid cancer in nodular thyroid disease. J Clin Endocrinol Metab. 2012;97:1134-45.

14. Boelaert K, Horacek J, Holder RL, Watkinson JC, Sheppard MC, Franklyn JA. Serum thyrotropin concentration as a novel predictor of malignancy in thyroid nodules investigated by fine-needle aspiration. J Clin Endocrinol Metab. 2006;91:4295-301.

15. Farid NR, Shi Y, Zou M. Molecular basis of thyroid cancer. Endocr Rev. 1994; 15:202-32.

16. American Thyroid Association (ATA) Guidelines Taskforce on Thyroid Nodules and Differentiated Thyroid Cancer, Cooper DS, Doherty GM Haugen BR, Kloos RT, Lee SL, et al. Revised American Thyroid Association management guidelines for patients with thyroid nodules and differentiated thyroid cancer. Thyroid. 2009;19:1167-214.

17. McLeod DS, Watters KF, Carpenter AD, Ladenson PW, Cooper DS, Ding EL. Thyrotropin and thyroid cancer diagnosis: a systematic review and doseresponse meta-analysis. J Clin Endocrinol Metab. 2012;97:2682-92.

18. Taylor PN, Razvi S, Pearce SH, Dayan CM. A review of the clinica consequences of variation in thyroid function within the reference range. J Clin Endocrinol Metab. 2013;98:3562-71.

19. Haymart MR, Repplinger DJ, Leverson GE, Elson DF, Sippel RS, Jaume JC, et al. Higher serum thyroid stimulating hormone level in thyroid nodule patients is associated with greater risks of differentiated thyroid cancer and advanced tumor stage. J Clin Endocrinol Metab. 2008;93:809-14.

20. Amphlett B, Lawson Z, Abdulrahman Jr GO, White C, Bailey R, Premawardhana LD, et al. Recent trends in the incidence, geographical distribution, and survival from thyroid cancer in Wales, 1985-2010. Thyroid. 2013;23:1470-8.

21. Chan DS, Okosieme OE. Recent trends in thyroid surgery in Wales. Surgeon. 2014;12:195-200.

22. Campanella P, lanni F, Rota CA, Corsello SM, Pontecorvi A. Quantification of cancer risk of each clinical and ultrasonographic suspicious feature of thyroid nodules: a systematic review and meta-analysis. Eur J Endocrinol. 2014;170:R203-11.

23. Reynolds RM, Weir J, Stockton DL, Brewster DH, Sandeep TC, Strachan MW. Changing trends in incidence and mortality of thyroid cancer in Scotland. Clin Endocrinol (Oxf). 2005;62:156-62.

24. Stojadinovic A, Peoples G, Libutti S, Henry L, Rberhardt J, Howard R, et al. Development of a clinical decision model for thyroid nodules. BMC Surg. 2009;9:12.

25. Liu Yl, Kamaya A, Desser TS, Rubin DL. A Bayesian network for differentiating benign from malignant thyroid nodules using sonographic and demographic features. Am J Roentgenol. 2011;196:W598-605.

26. Alfonso E, Sanabria A, Castillo M. Surgeons overestimate the risk of malignancy in thyroid nodules, evaluation of subjective estimates using a bayesian analysis. Biomedica. 2011;31:590-8.

27. Zahir S, Binesh F, Mirouliaei M, Khajeh E, Nashad S. Malignancy risk assessment in patients with thyroid nodules using classification and regression trees. J Thyroid Res. 2013;2013:983-53.

28. Maia FR, Matos PS, Silva B, Pallone A, Pavin E, Vassallo J, et al. Role of ultrasound, clinical and scintigraphic parameters to predict malignancy in thyroid nodule. Head Neck Oncol. 2011;3:17.
29. Banks ND, Kowalski J, Tsai HL, Semervell H, Tufano R, Dackiw AP, et al. A diagnostic predictor model for indeterminate for indeterminate or suspicious thyroid FNA samples. Thyroid. 2008;18:933-41.

30. Nixon IJ, Ganly I, Hann LE, Lin O, Yu C, Brandt S, et al. Nomogram for predicting malignancy in thyroid nodules using clinical, biochemical, ultrasonographic, and cytologic features. Surgery. 2010;148(6):1120-7.

31. Koike E, Noguchi S, Yamashita H, Murakami T, Ohshima A, Kawamoto H, et al. Ultrasonographic characteristics of thyroid nodules - prediction of malignancy. Arch Surg. 2001;136:334-7.

\section{Submit your next manuscript to BioMed Central and we will help you at every step:}

- We accept pre-submission inquiries

- Our selector tool helps you to find the most relevant journal

- We provide round the clock customer support

- Convenient online submission

- Thorough peer review

- Inclusion in PubMed and all major indexing services

- Maximum visibility for your research

Submit your manuscript at www.biomedcentral.com/submit 